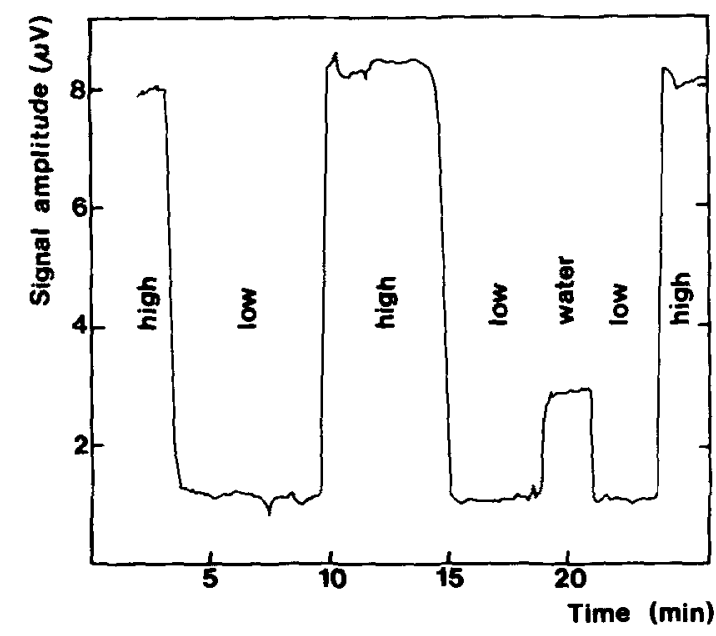

FIG. 2. Response from the flow-through cell for sudden changes in the concentration of the absorbing dye (Congo red). High concentration: $0.05 \mathrm{~g} / \mathrm{ml}$, low concentration: $0.01 \mathrm{~g} / \mathrm{ml}$.

There are at least three different kinds of applications of the flow-through technique. The first and most obvious is the study of flowing liquids (in, for instance, the process industry) where concentration of an absorbing substance in an opaque liquid can be monitored. The second application is to use the flow-through technique to obtain the average concentration of an absorbing substance in a large volume. Only samples within a certain distance from the window are analyzed. This distance is known as the thermal diffusion length $\mu$, equal to $(\kappa / \pi f \rho c)^{1 / 2}$, where $\kappa$ is the thermal conductivity, $\rho$ is the density, and $c$ is the specific heat of the sample, $f$ is the light modulation frequency. The thermal diffusion length is of the order of 10 to $100 \mu \mathrm{m}$ for most liquids and normal mod- ulation frequencies. The analyzed volume is thus rather small ( $1 \mu \mathrm{l}$ in the present setup) and it is sometimes desirable to analyze a larger volume to obtain a more representative measure. This can be done by circulating the sample through the cell. The third application is to use the flow-through technique to easily inject discrete samples in the cell. One sample volume is then injected each time. The flow is stopped and the analysis is performed. The cell is then cleaned by flowing, for instance, water through it and the next sample can be injected. Small sample volumes and fast analysis are possible if an automatic injection system is used.

The smallest absorption that can be detected depends mainly on the light intensity and the chopping frequency. With a strong light source and a low chopping frequency, the sensitivity of the photoacoustic technique is comparable with transmission measurements. On the other hand, it is possible to measure on very strongly absorbing samples. Absorption coefficients up to $10^{4} \mathrm{~cm}^{-1}$ are easily measured.

Several absorbing substances in a sample can be measured by using several wavelengths with different chopping frequencies. In this way it is possible to continuously measure the concentration of several absorbing substances in a flowing liquid. Experiments with two wavelengths have been performed with the present cell.

\footnotetext{
A. Rosencwaig, Photoacoustic Spectroscopy of Solids in Optoacoustic Spectroscopy and Detection, edited by Yoh-Han Pao, (Academic, New York, 1977).

2 P. Helander, J. Photoacoustics 1, 103 (1981).

3 P. Helander and I. Lundström (submitted to J. Photoacoustics).

${ }^{4} \mathrm{P}$. Helander (submitted to J. Photoacoustics).
}

\title{
Easily constructed high-vacuum valve
}

\section{S. L. Gilbert and C. E. Wieman}

Physics Department, University of Michigan, Ann Arbor, Michigan 48109

(Received 17 May 1982; accepted for publication 23 June 1982)

We present a design for a high-vacuum valve which can be built very quickly and easily. It has the additional feature that it is very thin.

PACS numbers: 07.30.Kf

We describe a high-vacuum valve which is extremely simple to construct. It is particularly well suited for use where a thin valve mechanism is needed to close a port in a large chamber.

As shown in Fig. 1, the valve itself is simply a $1 / 4-$ in.thick stainless-steel rectangle $6 \times 2 \frac{1}{2}$ in. with $1 / 4-20$ threaded holes at each end. For our particular case of a $1 \frac{1}{2}$ in. port to be sealed, a 2 -in. $O$ ring was seated in a groove in the face. A $1 / 4-20$ bolt is threaded through the left-hand hole (when facing the $\mathrm{O}$ ring) and a stop (two counter tightened nuts) is locked on its end. The smooth upper shaft of this bolt passes through a standard commercial $1 / 4$ in. quick connect feedthrough (ULTRA-TORR Male Connector, Cajon Company, Solon, Ohio) mounted in the chamber wall as shown in Fig. 2. When the valve is closed, a slightly shorter bolt comes through an identical feedthrough, and is screwed into the hole in the other end. A stop could be fastened to this bolt to prevent ac- 


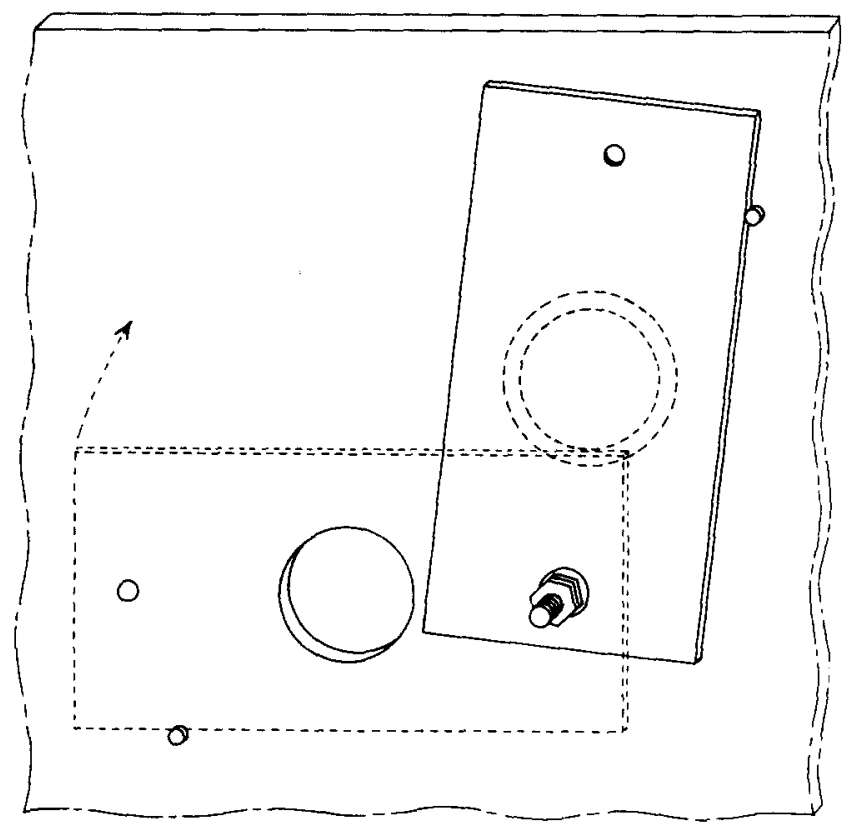

FIG. 1. Drawing of the valve as viewed from within the vacuum chamber. Solid figure is open position with O-ring groove on hidden face.

cidental removal under vacuum, but we have not found this necessary. Both bolts are tightened down, which presses the $\mathrm{O}$ ring against the smooth chamber wall.

To open the valve, the second bolt is disengaged and then the first is rotated counterclockwise. This pulls the $O$ ring away from the wall by moving the valve plate out along the threads to the stop. Another half turn pivots the plate away from the opening and then locks it against the

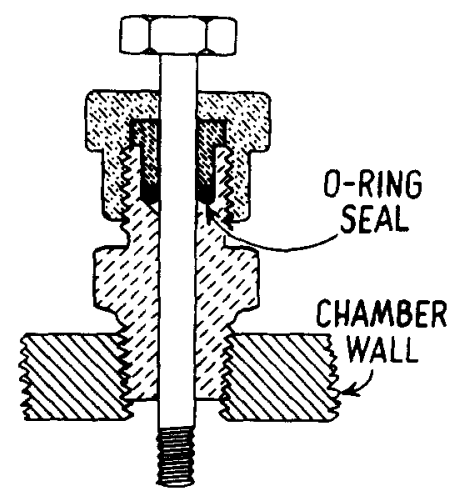

FIG. 2. Rotary feedthrough for the bolts. The $\mathrm{O}$ ring is lightly greased and the seal into the wall is made with Teflon pipethread tape.

stop. To close the valve the bolt is rotated clockwise which swings the plate back over the opening against a post set in the chamber wall, after which further turning pulls it against the wall and the second bolt is engaged. This is easily done under all conditions if the post has been mounted at the proper position.

This valve has worked well for 18 months of frequent use at pressures of $10^{-6}$ to $10^{-7}$ Torr. As well as the ease of construction and low profile (the entire mechanism inside the vacuum chamber is less than $5 / 8$ in. wide), we have found this design to avoid two common problems. There is no burst of air let into the system when opening or closing, as is frequently the case with valves that employ a shaft which slides through a feedthrough, and the O ring is not worn out rapidly as happens in valves where an $O$ ring slides over a surface.

One of us (SLG) is happy to acknowledge support from the Bendix Corporation Scholarship Fund. 